\title{
Infant fungal communities: current knowledge and research opportunities
}

Tonya L. Ward', Dan Knights ${ }^{1,2}$ and Cheryl A. Gale

\begin{abstract}
The microbes colonizing the infant gastrointestinal tract have been implicated in later-life disease states such as allergies and obesity. Recently, the medical research community has begun to realize that very early colonization events may be most impactful on future health, with the presence of key taxa required for proper immune and metabolic development. However, most studies to date have focused on bacterial colonization events and have left out fungi, a clinically important sub-population of the microbiota. A number of recent findings indicate the importance of host-associated fungi (the mycobiota) in adult and infant disease states, including acute infections, allergies, and metabolism, making characterization of early human mycobiota an important frontier of medical research. This review summarizes the current state of knowledge with a focus on factors influencing infant mycobiota development and associations between early fungal exposures and health outcomes. We also propose next steps for infant fungal mycobiome research, including longitudinal studies of mother-infant pairs while monitoring long-term health outcomes, further exploration of bacterium-fungus interactions, and improved methods and databases for mycobiome quantitation.
\end{abstract}

Keywords: Mycobiome, Fungi, Microbiome, Bacteria, Infant, Microbiota, Mycobiota, Development

\section{Background}

The beneficial role of microbial colonization to human health is becoming increasingly clear. Recent efforts to define a healthy microbiota show that the microbial communities inhabiting our bodies are diverse and complex, and that colonization dynamics during early life may have lasting impacts on adult health [1]. The term "microbiome" describes the community of microbes living on and within an organism using genetic analysis, usually within a particular niche or body site. Most literature discussing the microbiome, however, pertains only to the bacterial microbiota. Although bacteria constitute the majority of the non-host biomass of humans, they are not the only microorganisms contributing to the microbial ecosystem of the host. For example, human-associated fungi have been largely overlooked. On a cellular basis, approximately $0.1 \%$ of the microbes in the adult intestine are fungi, and these fungi are estimated to represent approximately 60 unique species [2,3]. Although fungi can be

\footnotetext{
* Correspondence: galex012@umn.edu

${ }^{3}$ Department of Pediatrics, University of Minnesota, 2450 Riverside Ave, Minneapolis, MN 55454, USA

Full list of author information is available at the end of the article
}

human pathogens, especially in association with underlying immunodeficiencies, many fungi are benign commensal inhabitants of human body niches and some have been shown to confer health benefits. For example, previous culture-based and targeted PCR approaches for characterizing the mycobiota have shown humans to be colonized with commensal fungi across multiple body sites $[4,5]$. Some species, such as Saccharomyces boulardii, have been shown to be effective at preventing and treating human gastrointestinal (GI) diseases (e.g., diarrhea, inflammatory bowel disease, irritable bowel syndrome) [6, 7]. Importantly, overgrowth of fungi leading to infections is more common in infants than adults and can result in significant morbidity and mortality in at-risk infants such as those born prematurely [8-10]. Thus, knowing what a healthy fungal microbiota (mycobiota) is composed of and what factors affect its establishment and maturation during infancy is important if we are to learn how early-life microbial communities affect pediatric and adult health.

With the advent of next-generation sequencing and introduction of low-cost bacterial community profiling approaches, such as $16 \mathrm{~S}$ rDNA amplicon sequencing, 
bacterial microbiomes can now be more easily and quickly characterized than ever before. In contrast, development of robust methods to characterize humanassociated mycobiota has lagged behind that for bacterial community characterization due to difficulties in sequencing low human-associated fungal biomass and fungal cultivation issues preventing reference sequence generation [11, 12]. Until recently, the majority of published fungal microbiota analyses have relied upon culturing fungi, a method that is less sensitive than sequencing-based approaches $[11,12]$. Because fungi are much less abundant than bacteria in most, if not all, human niches, a shotgun metagenomic sequencing approach for the characterization of fungal communities has not been productive. As such, PCR amplicon-based sequencing approaches have been developed and continue to be refined for mycobiome analyses. Genomic targets that have been utilized include the $18 \mathrm{~S}$ and $28 \mathrm{~S}$ rDNA sequences, and the internal transcribed spacer regions (ITS1 and ITS2) of the rDNA locus [12]. The ITS regions of the fungal genome are highly variable and capable of providing identification at the species level, but taxonomic characterization of fungi remains challenging as the reference databases available for fungi are far from comprehensive, with up to $20 \%$ of sequences annotated incorrectly [13]. Thus, mycobiome characterization may be biased by the marker gene region sequenced and the reference database used. Until improved reference databases are available and new marker gene sequences that more universally distinguish fungi are identified, methods combining broad DNA amplicon surveys with a targeted approach, such as quantitative PCR and/or culturing, will likely be needed to gain robust and accurate mycobiome characterization [14]. Given these challenges, throughout this review we note the particular approach employed by each study to characterize mycobiota.

Herein, we focus on the current state of mycobiota research in infants, including culture-based, targeted and broad survey-based genomic approaches, with a focus on two aspects of infant mycobiota research, namely factors influencing early-life mycobiota and potential links between early fungal exposures and health outcomes. Because research on the mycobiota of infants is limited, we consider the current state of fungal knowledge in the context of factors known to influence infant-associated bacterial microbiota, including mode of delivery, maternal mycobiota, feeding composition, environmental exposures (e.g., antibiotics), and gestational age at delivery [15-17]. Our findings are summarized in Figs. 1 and 2.

\section{Factors affecting infant mycobiota}

\section{Birth mode}

Whether an infant is born vaginally or through caesarean delivery (C-section) most drastically affects the composition

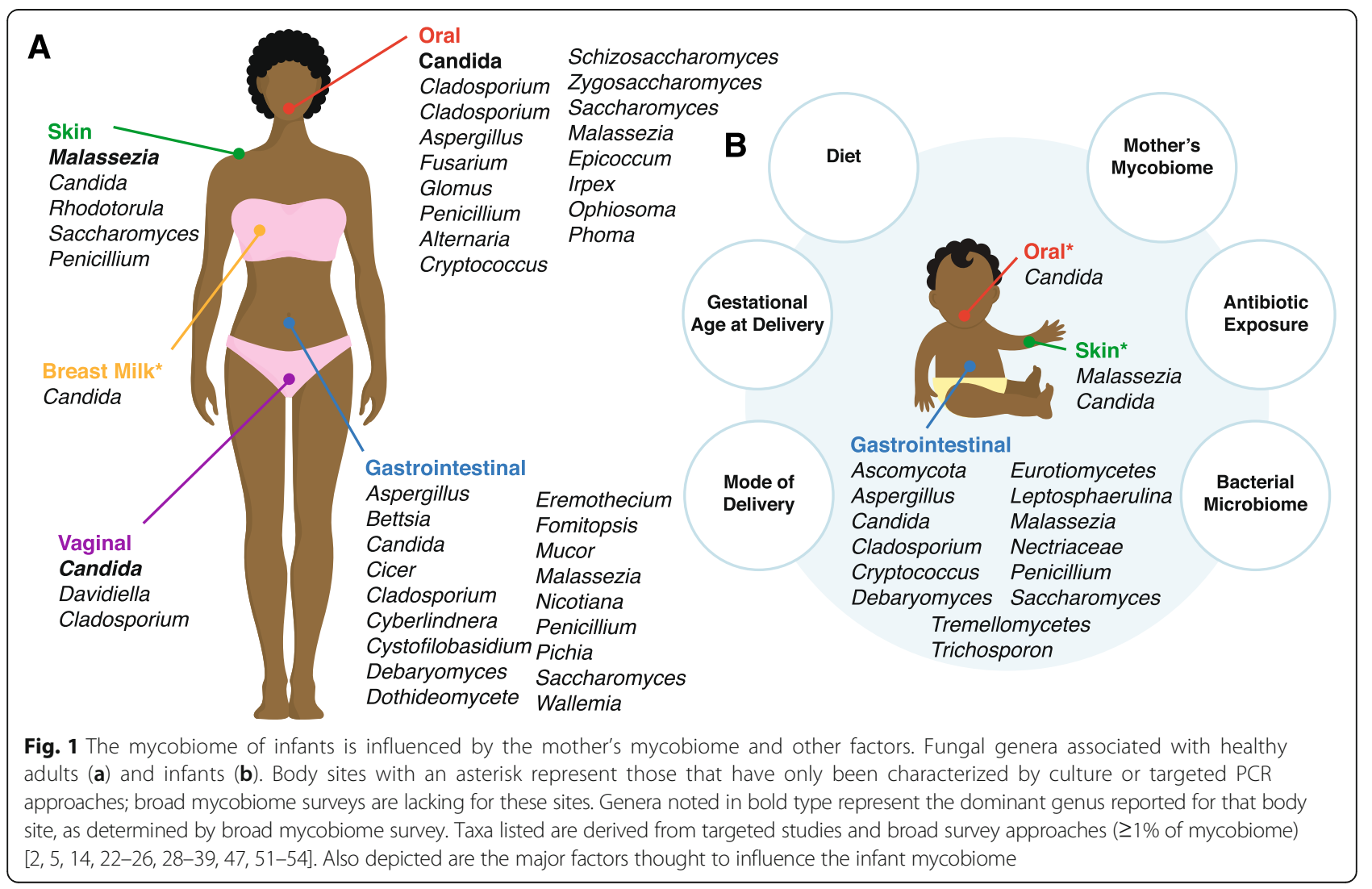




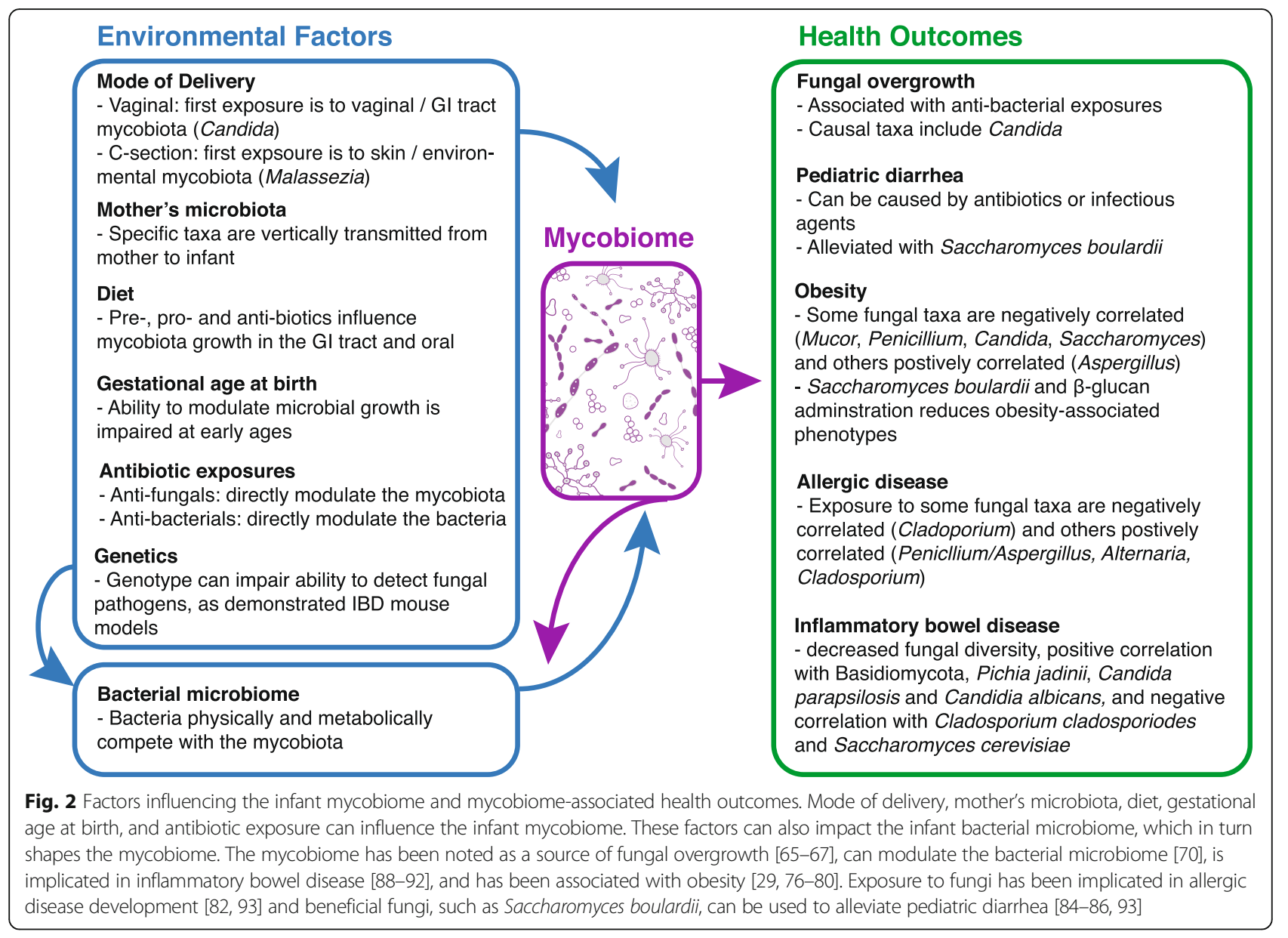

of their associated bacterial communities over the first 6 months of life [18-21]. For example, in a study of 98 mother-infant pairs, vaginally-born infants had less fecal Enterobacter, Haemophilus, Staphylococcus, Streptococcus, and Veillonella species and had increased Bacteroides, Bifidobacterium, Parabacteroides, and Escherichia compared to infants born by C-section [20]. In this same study, vertical transmission of the mother's fecal microbiota was likely the most significant contributor to the difference between vaginally and $\mathrm{C}$-section-born infants, as $72 \%$ of bacteria colonizing feces of vaginally-born infants were present in their mother's fecal microbiome, as compared to only $41 \%$ for C-section-born infants.

Vertical transmission of fungi from mother to infant has been most extensively studied with regard to the fungal species Candida albicans. In a study of very low birthweight infants, $24 \%$ of infants $(n=46)$ were colonized at a minimum of one site (oral cavity, rectum or groin) with $C$. albicans within 1 week of birth by the same C. albicans isolate present in either their mother's vagina, rectum, skin or mouth, as determined by culturing and DNA fingerprinting (Fig. 1) [22]. Given this example of vertical transmission of one fungal taxon, it is likely that other members of the maternal vaginal mycobiota are also transferred to the infant. Therefore, by better understanding fungi that inhabit the birth canal of the mother, we may gain insight into which fungal taxa may be transferred to the infant.

Historically, studies have focused on the characterization of one fungal genus within the female genital tract, Candida, due to its importance as a cause of infection (vaginitis). In a study of Candida colonization of the vagina of asymptomatic women without a history of vaginal candidiasis, $28.8 \%$ of women were positive for C. albicans by targeted PCR analysis, demonstrating that Candida is a normal commensal of the female genital tract (Fig. 1). Of note, only $6.6 \%$ of these women were positive for Candida by culture, highlighting the limitations of fungal community analysis using culture-based approaches [5]. In another screen of asymptomatic women without a history of vaginal candidiasis, $40 \%(n=52)$ of the sampled individuals carried at least one isolate of Candida ( $\sim 90 \%$ of which were C. albicans) in their genital tracts (vulva or vagina), as determined by DNA fingerprinting of cultured Candida [23]. To our knowledge, there has only been one published broad survey of the vaginal mycobiome using the ITS region [24]; in this survey of 
healthy women $(n=251)$, Candida was the predominant genus and was present in $68 \%$ of samples, followed by Davidiella, Cladosporium and other less abundant fungi (Fig. 1). Of note, a large number of sequences in this study were not classified into a fungal taxonomic group, likely due to the under-representation of fungal sequences in currently available databases, as well as inconsistencies in the taxonomic classification of fungi. Nevertheless, the vaginal mycobiota likely plays an important role in the early colonization events of vaginally born infants.

Apart from C. albicans, mother-infant transmission has not been well studied for fungi. In a recent survey of four infants and their mothers using universal $18 \mathrm{~S}$ rDNA primers [25], no amplicon was produced from DNA isolated from infant fecal samples at any time point, whereas the same samples were able to produce amplicons using universal $16 \mathrm{~S}$ primers for bacteria. Fecal DNA from the mothers in this study produced $18 \mathrm{~S}$ amplicons corresponding to Blastocystis (a parasite of the Stramenopile group), Saccharomyces, Candida, Nicotiana, and Cicer, among other fungal genera, leading the authors to conclude that the infant fecal samples contained no fungi. In contrast, in a study of very low birth weight infants, ITS2 amplicons were produced from the feces of the majority of infant samples (7 of 11) with the predominant genus being Saccharomyces ( $S$. cerevisiae) followed by Candida (C. albicans, C. glabrata, C. quercitrusa, C. diddensiae, C. parapsilosis, and C. tropicalis), Cladosporium (C. sphaerospermum and C. tenuissimum), and Cryptococcus (C. albidosimilas and $C$. podzolicus) [26]. Similarly, in a study of 11 infant fecal samples using fungal-specific PCR along with ITS2-based sequence analysis, fungi were observed in all samples, with $C$. albicans being the predominant species followed by $C$. parapsilosis, C. krusei, and Leptosphaerulina [14]. Penicillium, Aspergillus, Candida, Debaryomyces, Malassezia, Ascomycota, Eurotiomycetes, Tremellomycetes, Nectriaceae, and Trichosporon were also observed in an ITS1 survey of infants under 2 years of age, and the presence of many specific taxa was confirmed by culture-based methods [27]. A longitudinal study of 14 infants also showed fungi to be present in the feces of all infants, at most time points, at a density of $10^{4}-10^{6}$ rRNA genes/g of feces over the first 200 days of life [28]. This is in contrast to the $10^{9}-10^{10}$ bacterial rRNA genes/g of feces reported for the same samples [28]. Despite one study reporting a lack of fungi in the infant GI tract, we conclude that most infants do harbor GI fungi (Fig. 1). Although fecal fungal RNA appears to be less abundant than bacterial RNA, fungal cells are considerably larger (100-fold) than bacterial cells. Thus, fungi contribute a substantial biomass to the fecal microbiota.

The extent of transfer of fungal communities from mother to infant remains unclear, but the overlap of the infant and the adult GI mycobiome does support the hypothesis of vertical mycobiota transmission. For example, in adults, fungi were detected in all fecal samples of 96 individuals by ITS sequencing, and the most abundant genera were Saccharomyces, Candida, and Cladosporium [2]. Similarly, in healthy controls $(\mathrm{n}=12)$ from an ITS sequencebased survey of obese and lean individuals [29], Mucor, Candida, Penicillium, Wallemia, Bettsia, and Cladoporium were the predominant genera in feces, along with more minor members, and the healthy controls $(n=55)$ from a study of hepatitis-infected individuals were colonized with Saccharomyces, Candida, Aspergillus, Malassezia, Penicillium, and an uncharacterized fungus, as determined by $18 \mathrm{~S}$ restriction fragment length polymorphism (RFLP) analysis [30]. The adult GI mycobiome, as characterized by ITS1 sequencing, has also been shown to contain Candida, Penicillium, Aspergillus, Malassezia, Debaryomyces, Mucor, Eremothecium, Pichia, and Cyberlindnera (healthy controls, $n=29$ ) [31]. Further, Saccharomyces and Penicillium species were also detected by denaturing gradient gel electrophoresis and $18 \mathrm{~S}$ rDNA sequence analysis of fecal samples from healthy adults, along with other fungal genera [32], and Candida species predominated in a study of 45 adult fecal samples using ITS sequencing [33]. In the latter study, only two fungal taxa were shared in a majority of samples, indicating an absence of core GI tract mycobiota [33]. Additionally, the observation of instability across longitudinal samples in the study further indicated the lack of a core mycobiota, although further testing with a larger sample size should be performed to confirm this hypothesis [33]. The fungal taxa shared across the infant and adult studies include Candida, Saccharomyces, and Cladosporium, but given the limited number of studies in infants and their small sample sizes, there are likely more fungal taxa that overlap between the two groups (Fig. 1). The observation of shared taxa between adults and infants provides support for the hypothesis of vertical transmission of GI mycobiota from mother to infant.

In C-section born infants, the bacterial microbiota of the skin and GI tract are more similar to those of the mother's skin [21]. If this also holds true for the mycobiome, the skin and GI tract mycobiomes of C-section born infants would be expected to be dominated by Malassezia [34]. In a longitudinal study of infants that investigated skin colonization with Malassezia by Malassezia-specific PCR, two species (M. restricta and M. globosa, also observed on adult skin) were detected on the infant skin as early as the first day of life (89\%), and abundance levels increased to that observed in adults by day 30 of life (Fig. 1) [35]. In addition, in the same study, vertical transmission of Malassezia from the skin of mothers to that of infants was confirmed by genotyping of the intergenic spacer region located downstream of the ITS1 and 2 regions. The targeted approach used in this study, however, prevents us from 
determining the proportion of Malassezia relative to other mycobiome members, warranting further exploration of the infant skin mycobiota with broad survey approaches.

To better understand the infant skin mycobiota as a community, we can consider adult studies that employ culture-dependent and independent methods. For example, in a study of ITS1 sequences from 14 skin sites of 10 healthy adults, the skin mycobiome, with the exception of the feet, was dominated by the genus Malassezia [34], consistent with the findings of other studies using culture and targeted PCR approaches [34-39]. Other members of the skin mycobiome, found either on a small number of people or in low abundance, included Candida, Rhodotorula, Saccharomyces, and Penicillium, along with several much less abundant fungi $[34,37]$. For studies using ITS1 sequencing, however, it should be noted that such sequences are somewhat biased toward identification of basidiomycetous fungi, such as Malassezia, whereas ITS2 sequences favor identification of ascomycetous fungi such as most other humanassociated fungi, including Candida [40]. Thus, it is possible that the ITS1-based approach used in the study by Findley et al. [34] was not sensitive enough to fully detect Ascomycetes present in the samples. Nevertheless, the vertical transmission of Malassezia from mother to infant further supports the hypothesis that birth mode impacts the mycobiota of the infant. Based on the studies mentioned above, we can hypothesize that infants born vaginally would have a higher proportion of Candida in their mycobiome given the Candida-predominated birth canal, and potentially have a more diverse mycobiome in comparison to those born by $\mathrm{C}$-section given the exposure to the mother's varied fecal mycobiota. Conversely, we can hypothesize that $\mathrm{C}$-section infants, whose colonization source is often the mother's skin, would be colonized by relatively higher amounts of Malassezia (Fig. 2).

\section{Diet}

Diet composition, such as human milk or formula, strongly affects early infant bacterial microbiomes within the GI tract as well as in later infancy during the transition to solid foods [20, 41]. For example, breast-fed infants harbor more Bifidobacteria and Labctobacilli in their GI tracts in comparison to formula-fed infants [20,28], likely due to the endogenous microbiome of human milk and human milk factors, such as oligosaccharides and immune proteins, that modulate the growth of certain bacteria. In several studies, many bacterial genera found in human milk (Bifidobacterium, Bacteroides, Staphylococcus, Streptococcus, Pseudomonas, Lactobacillus, and others) were also found in infant fecal samples [42-44]. Additionally, prebiotics in breast milk, such as human milk oligosaccharides, promote the preferential expansion of certain taxa such as Bifidobacterium species [45, 46].
Although the human milk mycobiome has yet to be characterized, we know that some fungi, such as Candida species, are found in milk from mothers with mammary candidiasis $(67.4 \%, n=46)$ as well as in asymptomatic controls (79.1\%, $n=43$ ) (Fig. 1) [47]. Human milk oligosaccharides have also been demonstrated to impact fungal virulence in vitro by decreasing the ability of $C$. albicans to invade intestinal epithelial cells [48]. Thus, it is rational to hypothesize that human milk also influences the infant GI mycobiota, although this remains to be tested.

Diet also affects the oral bacterial microbiota of infants [49], and this likely holds true for the mycobiota. In infants, oral colonization with fungi has only been studied using culture-dependent methods, with Candida species appearing to be the predominant fungi, although this finding may be biased because these species grow most easily on certain growth media. In one study of 100 healthy infants, $12 \%$ were orally colonized with Candida by 4 weeks of age and colonization prevalence rates did not change over the first 6 months of life [50]. Other studies also show oral Candida colonization rates to be low at birth, but to rise over time to adult carriage rates within the first year of life [51,52]. The species of Candida currently reported as commensals of the oral cavity of infants include C. albicans, C. parapsilosis, C. krusei, C. guillermondii, C. geocandidum, and C. tropicalis [51, 52]. In adults, the oral mycobiota is reportedly diverse. For example, in one study using ITS2 sequencing to characterize oral mycobiomes, healthy individuals $(n=20)$ had a range of 9 to 23 fungal species within the oral cavity at a given time; the 'core' members of the oral community included Candida, Cladosporium, Aspergillus, Fusarium, Glomus, Penicillium, Alternaria, Cryptococcus, Ophiosoma, Phoma, Schizosaccharomyces, Zygosaccharomyces, and Saccharomyces [53]. Another study of adult saliva samples $(n=3)$ reported Malassezia as the dominant taxon, in addition to more minor mycobiome members such as Epicoccum or Irpex and others previously reported [54]. Therefore, given the diversity of the adult oral mycobiome, we can hypothesize that the oral mycobiome of infants likely contains other taxa in addition to Candida, and that the oral mycobiome of infants is likely dynamic, with changes especially noticeable as the infant transitions to a more adult-like diet (Fig. 2).

\section{Gestational age at delivery}

Gestational age at delivery has been shown to impact the bacterial microbiota, including initial community differences and the pace of bacterial community maturation, but differences are often resolved by 2 years of age, when the bacterial community has reached an adult-like state [55-57]. For infants born at an early gestational age, the health impact of intestinal fungi is particularly significant, as the incidence of invasive, 
systemic candidiasis in these infants is approximately $10 \%$, with an associated mortality rate of approximately $20 \%$ [58]. Susceptibility to invasive infection has been correlated with relative overgrowth of fungi, especially within the GI tract [8-10], as well as the presence of several predisposing clinical factors including a naïve immune system, bacterial dysbiosis due to antibiotic exposure, and use of parenteral nutrition, among other factors (Fig. 2) [59]. In an attempt to reduce rates of invasive candidiasis in this vulnerable population, prophylactic antifungals, such as nystatin and fluconazole, are often used in neonatal intensive care units. In particular, fluconazole has been shown to reduce Candida overgrowth at several body sites, including skin, the respiratory tract, and the GI tract, and its use has been associated with a decreased rate of invasive candidiasis in extremely low birth weight infants $[60,61]$. Recently, the enteral administration of bacterial and fungal probiotics, such as Lactobacillus reuteri, L. casei, L. rhamnosus, L. acidophilus, Streptococcus thermophilus, Bidiobacterium longum, B. bifidum, B. lactis, and S. boulardii, have also been used to reduce invasive candidiasis $[59,60]$; however, their efficacy remains unclear and their primary site of action is limited to the GI tract. Despite the administration of antifungals, fungal colonization still occurs in some infants, as demonstrated in a study of 11 extremely low birth weight infants, all of whom received an enteral anti-fungal treatment, nystatin, as well as antibacterial antibiotics [26]. Of these 11 infants, 7 produced ITS amplicons from their stool, with Saccharomycetales being the most abundant order, as well as Candida and Cryptococcus species. Thus, despite efforts to prevent fungal colonization, fungi maintain the ability to persist in the infant GI tract. In infants born at early gestational ages, beneficial fungi, such as $S$. boulardii, may help to regulate the growth of opportunistic fungal colonizers such as Candida. Additionally, given the association of bacterial dysbiosis with systemic candidiasis in infants born at an early gestational age, a robust bacterial community may play an important role in mycobiota regulation, as discussed below.

\section{Infant mycobiota health associations}

\section{Antibiotic exposure and fungal overgrowth}

The bacterial community of infants is significantly altered by exposure to antibiotics in both term and preterm infants $[16,62,63]$. For example, in a longitudinal study over the first 3 years of life, infants receiving multiple courses of antibiotics had immediate bacterial community changes following antibiotics and an overall less diverse fecal bacterial microbiome than those not given antibiotics [64]. Although most commonly used antibiotics do not directly act on fungi, anti-bacterial antibiotic exposure is associated with alterations to the mycobiota, such as increased rates of fungal colonization, fungal overgrowth, and changes in fungal community structure, as tested in mice [65]. In premature infants, exposure to third generation (broad-spectrum) cephalosporins is highly associated with an increased risk for both mucosal and invasive candidiasis [66], and in women, antibiotic exposure is associated with an increased incidence of fungal infections of the genitorurinary tract (Fig. 2) [67]. Recent studies have also reported that susceptibility to fungal overgrowth is primarily due to changes in the bacterial community following antibiotic exposure rather than immune status of the GI tract or the presence of specific fungal taxa $[68,69]$. The proposed mechanisms of fungal colonization regulation by bacteria include physical restriction, resource competition and the production of anti-fungal molecules $[68,69]$. Without these mechanisms of regulation, opportunistic fungi, such as Candida, can outgrow their occupied niche and initiate immune responses or cause acute infections, especially in immunocompromised individuals including premature infants.

The converse relationship that fungi affect bacterial colonization is also true, as recent animal experiments showed C. albicans colonization of the GI tract to be associated with altered bacterial repopulation following cefoperazone treatment, including increased Enterococcus faecalis levels and a lack of Lactobacillus recovery [70]. Specific bacterial and fungal taxa have also been shown to co-occur outside the scope of antimicrobial exposure. For example, in a study of stools from healthy adults, Candida was positively correlated with the presence of Prevotella, Ruminococcus, and Methanobrevibacter [2]. On adult skin, Malassezia was positively correlated with Corynebacterium, Prevotella, and Propionibacterium and negatively correlated with Pseudomonas, Acinetobacter, Streptococcus, and Enhydrobacter [71]. Additionally, certain fungal taxa are reported to regulate the growth of other fungi through the production of anti-fungal molecules. For example, the adult oral taxon, Pichia, is negatively correlated with the predominant oral taxon Candida and Pichia-produced factors isolated from growth media were able to prevent growth of Candida, Fusarium, and Aspergillus both in vitro and in mice [72]. Therefore, the fungal community is dynamic, self-regulating, and interacts with the bacterial community. Thus, future studies should include the analysis of both the bacterial and fungal components of the community in observational studies, as well as during experimental interventions (Fig. 2).

\section{Obesity}

In adults and children, obesity is associated with altered bacterial microbiota and, in infants, administration of antibiotics (known modulators of the bacterial community) is also associated with obesity later in life [73-75]. Similarly, fecal mycobiomes of obese and lean adults are 
reported to differ, with the genera Mucor and Penicillium negatively correlating with increased measures of adiposity and weight gain, and the genus Aspergillus positively correlating with increased measures of adiposity and weight gain [29]. In children, obesity has been associated with lower abundances of Candida and Saccharomyces species, as analyzed by DNA fingerprinting and quantitative PCR [76]. However, the role of early fungal colonization of infants and obesity outcomes has not yet been studied. We hypothesize that, similar to bacteria, some early-life fungi will be beneficial and some detrimental to short- and long-term metabolic health. For example, administration of $S$. boulardii and fungal-specific molecules ( $\beta$-glucan) to humans and animals have been shown to reduce obesity-related phenotypes [77-80]. Therefore, direct manipulation of obesityrelated fungal taxa in early life should be explored as a potential preventative strategy against obesity.

\section{Allergic disease}

Based on animal models and associations in humans, there are bacterial taxa that are associated with protection against allergic diseases later in life. For example, in a study of 319 infants, GI colonization with Faecalibacterium, Lachnospira, Veillonella, and Rothia was associated with a decreased risk of asthma and these same taxa were able to prevent airway inflammation in mice when used to colonize their GI tracts [81]. Whether there are key early-life fungal taxa that prevent allergic disease development remains unknown. However, a role for fungi in both allergy development and prevention was suggested by a study of 144 infants in whom exposure to airborne fungal spores from Penicillium/Aspergillus and Alternaria were positively associated with positive skin-prick tests for at least one allergen within a general allergy panel, whereas Cladosporium exposure was negatively associated with allergy development (Fig. 2). In addition, exposure to increased levels of environmental (bedroom) fungi during infancy were found to be negatively associated with laterchildhood development of wheeze and asthma, whereas increased indoor Aspergillus and outdoor Cladosporium levels were positively associated with a later increased incidence of rhinitis [82]. Thus, although the specific taxa and clinical outcomes involved need further exploration, there is strong evidence that early life exposure to certain fungal taxa contribute to allergic disease (Fig. 2).

\section{Pediatric diarrhea}

Diarrheal diseases are a significant cause of morbidity and mortality, accounting for approximately 760,000 deaths per year in children under the age of 5 [83]. The causes of diarrhea are varied, including antibioticassociated and infectious diarrhea, amongst others. $S$. boulardii has been evaluated as a treatment and preventative strategy for many types of diarrhea, with mostly positive outcomes $[84,85]$. For example, a metaanalysis of five clinical trials reported that $S$. boulardii is effective in preventing antibiotic-associated diarrhea in adults and children [86], and a separate meta-analysis also reported $S$. boulardii to be effective at reducing the duration of diarrhea in children with acute infectious gastroenteritis [87]. A third meta-analysis including 22 studies further concluded that $S$. boulardii can significantly reduce the duration of childhood diarrhea, whether caused by bacteria, viruses, or protozoa [85]. The mechanism whereby $S$. boulardii alleviates diarrhea is not yet clear, but animal models and in vitro work have shown $S$. boulardii to possess antimicrobial activities, inhibit bacterial toxins, promote barrier function of the intestinal epithelium, and elicit specific immune responses by the host [84]. Whether early exposure to $S$. boulardii is beneficial for the long-term health outcomes of infants remains to be tested, but the beneficial effect of $S$. boulardii on pediatric diarrhea is clear.

\section{Inflammatory bowel disease (IBD)}

The dysbiotic microbial communities associated with IBD are becoming more defined, including fungi associated with disease development. In pediatric patients (aged 3-21 years), there is a clear IBD-associated fungal signature as analyzed by ITS1 sequencing, including increased relative abundances of Pichia jadinii and Candida parapsilosis and a decreased relative abundance of Cladosporium cladosporiodes $(n=32)$, as well as an overall decrease in fungal diversity within the stool of IBD patients as compared to controls $(n=90)$ [88]. An additional study also found pediatric IBD patients to have a fungal community different than healthy controls, including a predominance of Basidiomycota, although only a small portion of colonic biopsies yielded fungal amplicons (8 of 37 samples) [89]. These results are supported by ITS 2 sequence analysis in a survey of adults with IBD that demonstrated an increased Basidiomycobiota/Ascomycota ratio, as well as an increased relative abundance of Candida albicans and decreased relative abundance of Saccharomyces cerevisiae in IBD patients $(n=235)$ as compared to controls $(n=38)$ [90]. While distinct gut fungal community structures are associated with IBD, it remains unclear as to whether microbial exposure or early colonization contributes to disease development. In a mouse model of chemically-induced colitis, fungi associated with IBD were capable of intestinal invasion only in animals deficient for the fungal pattern recognition receptor dectin-1 and not in wild-type animals. This suggests a causative role of host genetics, rather than a specific microbial community signature, in IBD [91]. Nevertheless, the fungal community appeared 
to contribute to disease severity, and future work regarding host-fungi interactions in early-life are needed to better understand IBD development and progression.

\section{Conclusion and future directions}

Although there is a relatively small number of broad mycobiome surveys for infants, by considering them in aggregate we can begin to answer certain important biological questions regarding early-life mycobiota development. For example, the use of targeted studies has shown the vertical transmission of specific fungal taxa, such and Candida and Malassezia, from mother to infant [22, 35], and this type of mycobiota inheritance is worth exploring for all mycobiome members. Additionally, broad surveys of infant feces have captured snapshots of the dynamic fungal community in early life, and have begun to shed light on other fungal taxa inhabiting infants $[14,25,26,28]$. The diversity and disease associations of adult mycobiota also provide further motivation to characterize infant mycobiota throughout development.

Future studies should focus on longitudinal tracking of the early-life mycobiota using mother-infant pairs while monitoring health outcomes, such as obesity, allergic disease, and acute fungal infections, well past the first year of life. The data generated from such studies would elucidate the impact of the mother's mycobiota, and other environmental factors, on the infant mycobiota, and would document temporal changes of the mycobiome throughout development. Additionally, given the known interactions between the bacteria and fungi, future studies should include the analysis of both bacteria and fungi to better characterize their structural and functional relationships, as well as their combined effects on health outcomes. Furthermore, more research is needed to address the methodological challenges in generating fungal microbiome data, including sampling of low fungal biomass, generation of comprehensive and reliable reference databases, and improvement of informatics methods tailored to classifying fungal taxonomy and mycobiota function.

\section{Acknowledgements}

Not applicable.

\section{Funding}

Not applicable.

Availability of data and material

Not applicable.

\section{Authors' contributions}

TW, CG and DK contributed in writing the manuscript. TW, CG and DK read and approved the final manuscript.

\section{Competing interests}

The authors declare that they have no competing interests.
Consent for publication

Not applicable.

Ethics approval and consent to participate

Not applicable.

\section{Author details}

${ }^{1}$ Biotechnology Institute, University of Minnesota, Saint Paul, MN, USA.

${ }^{2}$ Department of Computer Science and Engineering, University of Minnesota, Minneapolis, MN, USA. ${ }^{3}$ Department of Pediatrics, University of Minnesota, 2450 Riverside Ave, Minneapolis, MN 55454, USA.

Received: 30 August 2016 Accepted: 24 January 2017

Published online: 13 February 2017

References

1. Tamburini S, Shen N, Wu HC, Clemente JC. The microbiome in early life: implications for health outcomes. Nat Med. 2016;22:713-22.

2. Hoffmann C, Dollive S, Grunberg S, Chen J, Li H, Wu GD, et al. Archaea and fungi of the human gut microbiome: correlations with diet and bacterial residents. PLoS One. 2013;8, e66019.

3. Rajilić-Stojanović M, de Vos WM. The first 1000 cultured species of the human gastrointestinal microbiota. Fems Microbiol Rev. 2014;38:996-1047.

4. Balan P, B Gogineni S, Kumari NS, Shetty V, Lakshman Rangare A, L Castelino $R$, et al. Candida carriage rate and growth characteristics of saliva in diabetes mellitus patients: A case-control study. J Dent Res Dent Clin Dent Prospects. 2015;9:274-9.

5. Giraldo P, von Nowaskonski A, Gomes FA, Linhares I, Neves NA, Witkin SS. Vaginal colonization by Candida in asymptomatic women with and without a history of recurrent vulvovaginal candidiasis. Obstet Gynecol. 2000;95:413-6.

6. Moré MI, Swidsinski A. Saccharomyces boulardii CNCM I-745 supports regeneration of the intestinal microbiota after diarrheic dysbiosis - a review. Clin Exp Gastroenterol. 2015;8:237-55.

7. Zanello G, Meurens F, Berri M, Salmon H. Saccharomyces boulardii effects on gastrointestinal diseases. Curr Issues Mol Biol. 2009;11:47-58.

8. Coates EW, Karlowicz MG, Croitoru DP, Buescher ES. Distinctive distribution of pathogens associated with peritonitis in neonates with focal intestinal perforation compared with necrotizing enterocolitis. Pediatrics. 2005;116:e241-6.

9. Ragouilliaux CJ, Keeney SE, Hawkins HK, Rowen JL. Maternal factors in extremely low birth weight infants who develop spontaneous intestinal perforation. Pediatrics. 2007;120:e1458-64.

10. Saiman L, Ludington E, Pfaller M, Rangel-Frausto S, Wiblin RT, Dawson J, et al. Risk factors for candidemia in Neonatal Intensive Care Unit patients. Pediatr Infect Dis J. 2000;19:319-24.

11. Suhr MJ, Hallen-Adams HE. The human gut mycobiome: pitfalls and potentials-a mycologist's perspective. Mycologia. 2015;107:1057-73.

12. Underhill DM, Iliev ID. The mycobiota: interactions between commensal fungi and the host immune system. Nat Rev Immunol. 2014:14:405-16.

13. Nilsson RH, Ryberg M, Kristiansson E, Abarenkov K, Larsson K-H, Kõljalg U. Taxonomic reliability of DNA sequences in public sequence databases: A fungal perspective. PLoS One. 2006;1, e59.

14. Heisel T, Podgorski H, Staley CM, Knights D, Sadowsky MJ, Gale CA. Complementary amplicon-based genomic approaches for the study of fungal communities in humans. PLoS One. 2015;10, e0116705.

15. Lim ES, Wang D, Holtz LR. The bacterial microbiome and virome milestones of infant development. Trends Microbiol. 2016;24(10):801-10.

16. Vangay P, Ward T, Gerber JS, Knights D. Antibiotics, pediatric dysbiosis, and disease. Cell Host Microbe. 2015;17:553-64.

17. Mueller NT, Bakacs E, Combellick J, Grigoryan Z, Dominguez-Bello MG. The infant microbiome development: mom matters. Trends Mol Med. 2015;21:109-17.

18. Martin R, Makino H, Yavuz AC, Ben-Amor K, Roelofs M, Ishikawa E, et al. Earlylife events, including mode of delivery and type of feeding, siblings and gender, shape the developing gut microbiota. PLoS One. 2016;11, e0158498.

19. Rutayisire E, Huang K, Liu Y, Tao F. The mode of delivery affects the diversity and colonization pattern of the gut microbiota during the first year of infants' life: a systematic review. BMC Gastroenterol. 2016;16:86

20. Bäckhed F, Roswall J, Peng Y, Feng Q, Jia H, Kovatcheva-Datchary P, et al. Dynamics and stabilization of the human gut microbiome during the first year of life. Cell Host Microbe. 2015;17:690-703.

21. Dominguez-Bello MG, Costello EK, Contreras M, Magris M, Hidalgo G, Fierer $\mathrm{N}$, et al. Delivery mode shapes the acquisition and structure of the initial 
microbiota across multiple body habitats in newborns. Proc Natl Acad Sci U S A. 2010;107:11971-5.

22. Bliss JM, Basavegowda KP, Watson WJ, Sheikh AU, Ryan RM. Vertical and horizontal transmission of Candida albicans in very low birth weight infants using DNA fingerprinting techniques. Pediatr Infect Dis J. 2008;27:231-5.

23. Soll DR, Galask R, Schmid J, Hanna C, Mac K, Morrow B. Genetic dissimilarity of commensal strains of Candida spp. carried in different anatomical locations of the same healthy women. J Clin Microbiol. 1991;29:1702-10.

24. Drell T, Lillsaar T, Tummeleht L, Simm J, Aaspõllu A, Väin E, et al. Characterization of the vaginal micro- and mycobiome in asymptomatic reproductive-age Estonian women. PLoS One. 2013;8, e54379.

25. Pandey PK, Siddharth J, Verma P, Bavdekar A, Patole MS, Shouche YS. Molecular typing of fecal eukaryotic microbiota of human infants and their respective mothers. J Biosci. 2012;37:221-6.

26. LaTuga MS, Ellis JC, Cotton CM, Goldberg RN, Wynn JL, Jackson RB, et al. Beyond bacteria: a study of the enteric microbial consortium in extremely low birth weight infants. PLoS One. 2011;6, e27858.

27. Strati F, Di Paola M, Stefanini I, Albanese D, Rizzetto L, Lionetti P, et al. Age and gender affect the composition of fungal population of the human gastrointestinal tract. Front Microbiol. 2016;7:1227.

28. Palmer C, Bik EM, DiGiulio DB, Relman DA, Brown PO. Development of the human infant intestinal microbiota. PLoS Biol. 2007;5, e177.

29. Mar Rodríguez M, Pérez D, Javier Chaves F, Esteve E, Marin-Garcia P, Xifra G, et al. Obesity changes the human gut mycobiome. Sci Rep. 2015;5:14600.

30. Chen Y, Chen Z, Guo R, Chen N, Lu H, Huang S, et al. Correlation between gastrointestinal fungi and varying degrees of chronic hepatitis $B$ virus infection. Diagn Microbiol Infect Dis. 2011;70:492-8.

31. Strati F, Cavalieri D, Albanese D, De Felice C, Donati C, Hayek J, et al. Altered gut microbiota in Rett syndrome. Microbiome. 2016;4:41

32. Ott SJ, Kühbacher T, Musfeldt M, Rosenstiel P, Hellmig S, Rehman A, et al. Fungi and inflammatory bowel diseases: alterations of composition and diversity. Scand J Gastroenterol. 2008:43:831-41.

33. Hallen-Adams HE, Kachman SD, Kim J, Legge RM, Martínez I. Fungi inhabiting the healthy human gastrointestinal tract: a diverse and dynamic community. Fungal Ecol. 2015;15:9-17.

34. Findley K, Oh J, Yang J, Conlan S, Deming C, Meyer JA, et al. Topographic diversity of fungal and bacterial communities in human skin. Nature. 2013;498:367-70.

35. Nagata $R$, Nagano $H$, Ogishima D, Nakamura $Y$, Hiruma M, Sugita $T$. Transmission of the major skin microbiota, Malassezia, from mother to neonate. Pediatr Int. 2012;54:350-5.

36. Park HK, Ha M-H, Park S-G, Kim MN, Kim BJ, Kim W. Characterization of the fungal microbiota (mycobiome) in healthy and dandruff-afflicted human scalps. PLoS One. 2012;7, e32847.

37. Zhang E, Tanaka T, Tajima M, Tsuboi R, Nishikawa A, Sugita T. Characterization of the skin fungal microbiota in patients with atopic dermatitis and in healthy subjects. Microbiol Immunol. 2011;55:625-32.

38. Paulino LC, Tseng C-H, Blaser MJ. Analysis of Malassezia microbiota in healthy superficial human skin and in psoriatic lesions by multiplex realtime PCR. FEMS Yeast Res. 2008:8:460-71.

39. Paulino LC, Tseng C-H, Strober BE, Blaser MJ. Molecular analysis of fungal microbiota in samples from healthy human skin and psoriatic lesions. J Clin Microbiol. 2006:44:2933-41.

40. Bellemain E, Carlsen T, Brochmann C, Coissac E, Taberlet P, Kauserud H. ITS as an environmental DNA barcode for fungi: an in silico approach reveals potential PCR biases. BMC Microbiol. 2010;10:189.

41. Koenig JE, Fricker AD, Scalfon N, Kotman N, Stombaugh J, Knight R, et al. Succession of microbial consortia in the developing infant gut microbiome. Proc Natl Acad Sci U S A. 2011;108:4578-85

42. Jost $T$, Lacroix C, Braegger CP, Rochat F, Chassard C. Vertical motherneonate transfer of maternal gut bacteria via breastfeeding. Environ Microbiol. 2014;16:2891-904.

43. Cabrera-Rubio R, Collado MC, Laitinen K, Salminen S, Isolauri E, Mira A. The human milk microbiome changes over lactation and is shaped by maternal weight and mode of delivery. Am J Clin Nutr. 2012;96:544-51.

44. Hunt KM, Foster JA, Forney LJ, Schütte UME, Beck DL, Abdo Z, et al. Characterization of the diversity and temporal stability of bacterial communities in human milk. PLoS One. 2011;6, e21313.

45. De Leoz MLA, Kalanetra KM, Bokulich NA, Strum JS, Underwood MA German JB, et al. Human milk glycomics and gut microbial genomics in infant feces show a correlation between human milk oligosaccharides and gut microbiota: a proof-of-concept study. J Proteome Res. 2015;14:491-502.
46. Asakuma S, Hatakeyama E, Urashima T, Yoshida E, Katayama T, Yamamoto K, et al. Physiology of consumption of human milk oligosaccharides by infant gut-associated bifidobacteria. J Biol Chem. 2011;286:34583-92.

47. Mutschlechner W, Karall D, Hartmann C, Streiter B, Baumgartner-Sigl S, Orth-Höller D, et al. Mammary candidiasis: molecular-based detection of Candida species in human milk samples. Eur J Clin Microbiol Infect Dis. 2016;35:1309-13.

48. Gonia S, Tuepker M, Heisel T, Autran C, Bode L, Gale CA. Human milk oligosaccharides inhibit Candida albicans invasion of human premature intestinal epithelial cells. J Nutr. 2015;145:1992-8.

49. Holgerson PL, Vestman NR, Claesson R, Öhman C, Domellöf M, Tanner AC, et al. Oral microbial profile discriminates breastfed from formula-fed Infants. J Pediatr Gastroenterol Nutr. 2013;56:127-36.

50. Stecksén-Blicks C, Granström E, Silfverdal SA, West CE. Prevalence of oral Candida in the first year of life. Mycoses. 2015;58:550-6.

51. Kleinegger CL, Lockhart SR, Vargas K, Soll DR. Frequency, intensity, species, and strains of oral Candida vary as a function of host age. J Clin Microbiol. 1996:34:2246-54.

52. Russell C, Lay KM. Natural history of Candida species and yeasts in the oral cavities of infants. Arch Oral Biol. 1973;18:957-62.

53. Ghannoum MA, Jurevic RJ, Mukherjee PK, Cui F, Sikaroodi M, Naqvi A, et al. Characterization of the oral fungal microbiome (mycobiome) in healthy individuals. PLoS Pathog. 2010;6, e1000713.

54. Dupuy AK, David MS, Li L, Heider TN, Peterson JD, Montano EA, et al. Redefining the human oral mycobiome with improved practices in amplicon-based taxonomy: discovery of Malassezia as a prominent commensal. PLoS One. 2014;9, e90899.

55. Ardissone AN, de la Cruz DM, Davis-Richardson AG, Rechcigl KT, Li N, Drew $\mathrm{JC}$, et al. Meconium microbiome analysis identifies bacteria correlated with premature birth. PLoS One. 2014;9, e90784.

56. La Rosa PS, Warner BB, Zhou Y, Weinstock GM, Sodergren E, Hall-Moore CM, et al. Patterned progression of bacterial populations in the premature infant gut. Proc Natl Acad Sci U S A. 2014;111:12522-7.

57. Moles L, Gómez M, Jiménez E, Fernández L, Bustos G, Chaves F, et al. Preterm infant gut colonization in the neonatal ICU and complete restoration 2 years later. Clin Microbiol Infect. 2015;21:936. e1-10.

58. Greenberg RG, Benjamin DK. Neonatal candidiasis: diagnosis, prevention, and treatment. J Infect. 2014;69 Suppl 1:S19-22.

59. Kumar S, Singhi S. Role of probiotics in prevention of Candida colonization and invasive candidiasis. J Matern Fetal Neonatal Med. 2016;29:818-9.

60. Manzoni P, Mostert M, Castagnola E. Update on the management of Candida infections in preterm neonates. Arch Dis Child Fetal Neonatal Ed. 2015;100:F454-9.

61. Kaufman DA, Gurka MJ, Hazen KC, Boyle R, Robinson M, Grossman LB. Patterns of fungal colonization in preterm infants weighing less than 1000 grams at birth. Pediatr Infect Dis J. 2006;25:733-7.

62. Gibson MK, Wang B, Ahmadi S, Burnham C-AD, Tarr PI, Warner BB, et al. Developmental dynamics of the preterm infant gut microbiota and antibiotic resistome. Nat Microbiol. 2016;1:16024.

63. Langdon A, Crook N, Dantas G. The effects of antibiotics on the microbiome throughout development and alternative approaches for therapeutic modulation. Genome Med. 2016;8:39.

64. Yassour M, Vatanen T, Siljander H, Hämäläinen A-M, Härkönen T, Ryhänen SJ, et al. Natural history of the infant gut microbiome and impact of antibiotic treatment on bacterial strain diversity and stability. Sci Transl Med. 2016;8:343ra81.

65. Dollive $S$, Chen $Y-Y$, Grunberg S, Bittinger $K$, Hoffmann $C$, Vandivier $L$, et al. Fungi of the murine gut: episodic variation and proliferation during antibiotic treatment. PLoS One. 2013;8, e71806.

66. Kelly MS, Benjamin DK, Smith PB. The epidemiology and diagnosis of invasive Candidiasis among premature infants. Clin Perinatol. 2015;42:105-17.

67. Achkar JM, Fries BC. Candida infections of the genitourinary tract. Clin Microbiol Rev. 2010:23:253-73.

68. Shankar J, Solis NV, Mounaud S, Szpakowski S, Liu H, Losada L, et al. Using Bayesian modelling to investigate factors governing antibiotic-induced Candida albicans colonization of the Gl tract. Sci Rep. 2015;5:8131.

69. Helstrom PB, Balish E. Effect of oral tetracycline, the microbial flora, and the athymic state on gastrointestinal colonization and infection of BALB/c mice with Candida albicans. Infect Immun. 1979;23:764-74.

70. Mason KL, Erb Downward JR, Mason KD, Falkowski NR, Eaton KA, Kao JY, et al. Candida albicans and bacterial microbiota interactions in the cecum during recolonization following broad-spectrum antibiotic therapy. Infect Immun. 2012:80:3371-80. 
71. Leung MHY, Chan KCK, Lee PKH. Skin fungal community and its correlation with bacterial community of urban Chinese individuals. Microbiome. 2016;4:46.

72. Mukherjee PK, Chandra J, Retuerto M, Sikaroodi M, Brown RE, Jurevic R, et al. Oral mycobiome analysis of HIV-infected patients: identification of Pichia as an antagonist of opportunistic fungi. PLoS Pathog. 2014;10, e1003996.

73. Turnbaugh PJ, Hamady M, Yatsunenko T, Cantarel BL, Duncan A, Ley RE, et al. A core gut microbiome in obese and lean twins. Nature. 2009;457:480-4.

74. Bailey L, Forrest CB, Zhang P, Richards TM, Livshits A, DeRusso PA. Association of antibiotics in infancy with early childhood obesity. JAMA Pediatr. 2014;168:1063-9.

75. Riva A, Borgo F, Lassandro C, Verduci E, Morace G, Borghi E, et al. Pediatric obesity is associated with an altered gut microbiota and discordant shifts in Firmicutes populations. Environ Microbiol. 2016. doi:10.1111/1462-2920.13463.

76. Borgo F, Verduci E, Riva A, Lassandro C, Riva E, Morace G, et al. Relative abundance in bacterial and fungal gut microbes in obese children: A case control study. Child Obes. 2016. Ahead of print.

77. Everard A, Matamoros S, Geurts L, Delzenne NM, Cani PD. Saccharomyces boulardii administration changes gut microbiota and reduces hepatic steatosis, low-grade inflammation, and fat mass in obese and type 2 diabetic db/db mice. MBio. 2014;5:e01011-4.

78. de Araújo TV, Andrade EF, Lobato RV, Orlando DR, Gomes NF, de Sousa RV, et al. Effects of beta-glucans ingestion (Saccharomyces cerevisiae) on metabolism of rats receiving high-fat diet. J Anim Physiol Anim Nutr. 2016. doi:10.1111/jpn.12452.

79. Neyrinck AM, Possemiers S, Verstraete W, De Backer F, Cani PD, Delzenne NM. Dietary modulation of clostridial cluster XIVa gut bacteria (Roseburia spp.) by chitin-glucan fiber improves host metabolic alterations induced by high-fat diet in mice. J Nutr Biochem. 2012;23:51-9.

80. Nicolosi R, Bell SJ, Bistrian BR, Greenberg I, Forse RA, Blackburn GL. Plasma lipid changes after supplementation with beta-glucan fiber from yeast. Am J Clin Nutr. 1999:70:208-12

81. Arrieta M-C, Stiemsma LT, Dimitriu PA, Thorson L, Russell S, Yurist-Doutsch S, et al. Early infancy microbial and metabolic alterations affect risk of childhood asthma. Sci Transl Med. 2015;7:307ra152.

82. Behbod B, Sordillo JE, Hoffman EB, Datta S, Webb TE, Kwan DL, et al Asthma \& allergy development: contrasting influences of yeasts \& other fungal exposures. Clin Exp Allergy J. 2015:45:154-63.

83. World Health Organization. Diarrhoeal disease. http://www.who.int/ mediacentre/factsheets/fs330/en/.

84. Kelesidis T, Pothoulakis C. Efficacy and safety of the probiotic Saccharomyces boulardii for the prevention and therapy of gastrointestinal disorders. Ther Adv Gastroenterol. 2012;5:111-25.

85. Feizizadeh S, Salehi-Abargouei A, Akbari V. Efficacy and safety of Saccharomyces boulardii for acute diarrhea. Pediatrics. 2014;134:e176-91.

86. Szajewska H, Mrukowicz J. Meta-analysis: non-pathogenic yeast Saccharomyces boulardii in the prevention of antibiotic-associated diarrhoea. Aliment Pharmacol Ther. 2005;22:365-72.

87. Szajewska H, Canani RB, Guarino A, Hojsak I, Indrio F, Kolacek S, et al. Probiotics for the prevention of antibiotic-associated diarrhea in children. $J$ Pediatr Gastroenterol Nutr. 2016;62:495-506.

88. Chehoud C, Albenberg LG, Judge C, Hoffmann C, Grunberg S, Bittinger K, et al. Fungal signature in the gut microbiota of pediatric patients with inflammatory bowel disease. Inflamm Bowel Dis. 2015;21:1948-56.

89. Mukhopadhya I, Hansen R, Meharg C, Thomson JM, Russell RK, Berry SH, et al. The fungal microbiota of de-novo paediatric inflammatory bowel disease. Microbes Infect. 2015;17:304-10.

90. Sokol H, Leducq V, Aschard H, Pham H-P, Jegou S, Landman C, et al. Fungal microbiota dysbiosis in IBD. Gut. 2016. doi:10.1136/gutjnl-2015-310746.

91. Iliev ID, Funari VA, Taylor KD, Nguyen Q, Reyes CN, Strom SP, et al. Interactions between commensal fungi and the C-type lectin receptor dectin-1 influence colitis. Science. 2012;336:1314-7.

92. Osborne M, Reponen T, Adhikari A, Cho S-H, Grinshpun SA, Levin L, et al. Specific fungal exposures, allergic sensitization, and rhinitis in infants. Pediatr Allergy Immunol. 2006;17:450-7.

93. Szajewska H, Skórka A, Dylag M. Meta-analysis: Saccharomyces boulardii for treating acute diarrhoea in children. Aliment Pharmacol Ther. 2007;25:257-64.

\section{Submit your next manuscript to BioMed Central and we will help you at every step:}

- We accept pre-submission inquiries

- Our selector tool helps you to find the most relevant journal

- We provide round the clock customer support

- Convenient online submission

- Thorough peer review

- Inclusion in PubMed and all major indexing services

- Maximum visibility for your research

Submit your manuscript at www.biomedcentral.com/submit 